\title{
ELECTRICAL DISCHARGE FROM A POINT TO A PLANE.
}

\author{
By O. Hovda.
}

I. E. Warburg ${ }^{1}$ investigated the effect of distance between a point and a plane upon the current flowing for a given potential, using distances up to $7 \mathrm{~cm}$. In the following experiments the results have been extended to much greater distances, and the variation with distance of the minimum potential required to produce a discharge has been investigated as well as the variation of the current with the potential at the different distances. The potential gradient between the point and the plane has also been investigated and a relation found between its value at any place and the current flowing.

2. The arrangement of the apparatus and method of taking the readings were similar to those used by $\mathrm{J}$. Zeleny, ${ }^{2}$ except that a resistance of carbon-ruled paper ${ }^{3}$ was used to regulate the voltage from the static machine. The plane used consisted of a large circular zinc plate, 330 $\mathrm{cm}$. in diameter. The discharge point was the hemispherical end of a platinum wire, $1.75 \mathrm{~cm}$. long and $.013 \mathrm{~cm}$. in diameter, supported at the end of a brass rod $75 \mathrm{~cm}$. long made up of sections of increasing diameter for the sake of rigidity. Because of the large size of the apparatus the experiment was necessarily carried on in the open air of the room. The results are reduced to $22^{\circ} \mathrm{C}$. and $74 \mathrm{~cm}$. pressure according to the laws of variation in these respects found by J. Zeleny. ${ }^{4}$

3. Minimum Potential.-Most of the observations were taken with a galvanometer joined to the plane to show when the current started to flow, but in some cases this was replaced by a sensitive electroscope without any change in the results. The results for the starting potential for both positive and negative discharges are represented by the curves in Fig. 1. Each circle represents the average of several readings taken on different days. It is seen that for the smaller distances the minimum potential increases quite rapidly with increase in distance, but changes very slowly with the larger distances. It appears probable therefore that for all very large distances there would be a constant minimum potential. This may have some application in the consideration of the

1 E. Warburg, Ann. der Phys., 67, p. 69, 1899.

2 Phys. Rev., Vol. XXV., p. 305, 1907.

${ }^{3}$ F. Aust, Physik. Zeitschr., I2, p. 732, I9II.

${ }^{4}$ Loc. cit., p. 326. 
[VoL. XXXIV.

discharge produced from a lightning-rod point by a cloud. It is quite likely that the discharge begins when there is a certain definite electrical density on the point, and this density is reached at a lower voltage when an earthed plane is situated near by owing to the condenser effect; but

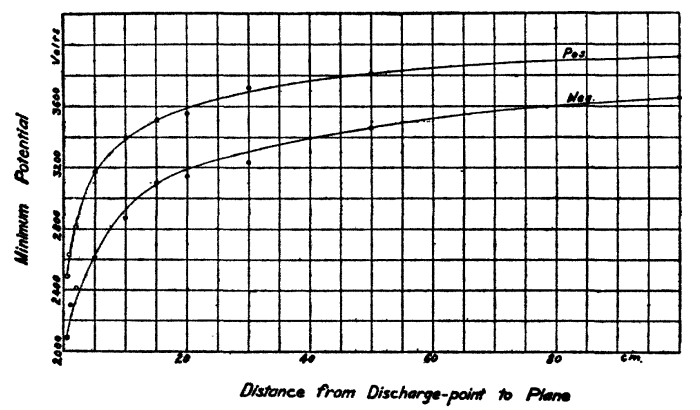

Fig. 1.

when the distance is considerable a further increase produces but a slight change in the electrical density due to a given potential. For both kinds of discharge an equation of the form

$$
M=a-\frac{b}{c+\sqrt{D}}
$$

was found to represent quite closely the dependence of minimum potential on distance. Expressing $D$ in centimeters and $M$ in volts, the experimental results are represented by the following equations:

For positive discharge,

For negative discharge,

$$
M=4260-\frac{3740}{1.17+\sqrt{D}} .
$$

$$
M=4290-\frac{8400}{3.06+\sqrt{D}} .
$$

The approximate equality of the constant $a$ for the two kinds of discharge is an indication that the two starting potentials for very large distances would be nearly identical.

4. Currents - In order to approximate to an infinite plane, the receiving surface must be large when the distance between the point and the plane becomes considerable. According to a preliminary experiment a plane of the size used $(330 \mathrm{~cm}$.) should receive as much current as an infinite plane for distances up to $54 \mathrm{~cm}$. between the point and the plane, while if the latter distance be as much as $100 \mathrm{~cm}$., the current should not be more than 4 per cent. less than that received by an 
infinite plane. The variation in current with voltage for the various distances between the point and the plane is shown by the curves of Figs. 2 and 3. These represent average results of three, and, in some

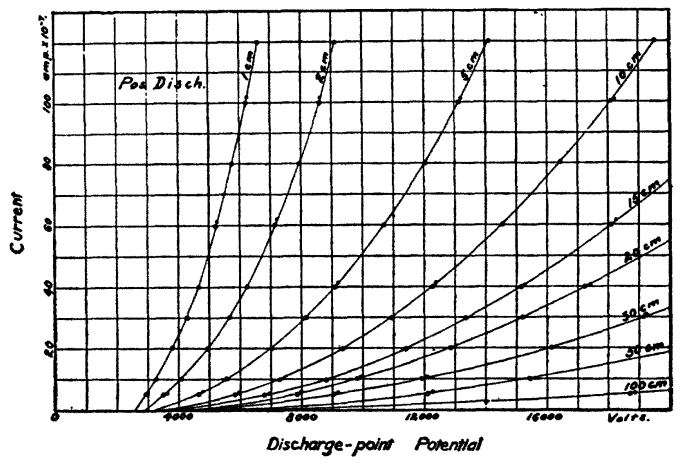

Fig. 2.

cases, four independent determinations taken at different times. It is seen that for the short distances the current increases rapidly with increase in voltage, while at the larger distances the current increases very slowly.

The results for all distances cannot be represented even roughly by an equation of the form given by Warburg, ${ }^{1}$ who used distances up to 7 centimeters only. The experimental values of the current $I$ may be

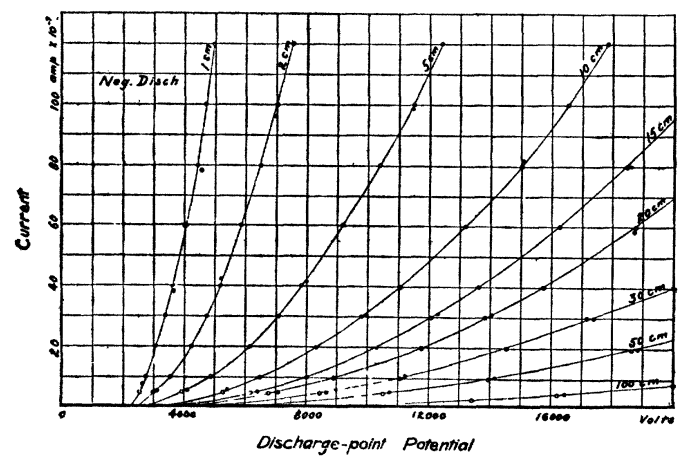

Fig. 3.

represented, however, in terms of the voltage $V$, minimum potential $M$, and distance $D$, by the following equations:

For positive discharge,

$$
I=\frac{43 \mathrm{I} \times 10^{-15} V(V-M)}{\left(D+.0000589 D^{3}\right)^{1.08}} \mathrm{amp} .
$$

${ }^{1}$ Loc. cit., p. 83 . 
For negative discharge,

$$
I=\frac{710 \times 10^{-15} V(V-M)}{\left(D-.00692 D^{2}+.0000659 D^{3}\right)^{1.24}} \text { amp. }
$$

The points shown by the filled circles in Figs. 2 and 3 , indicate the degree of agreement with the experimental results which are represented by open circles. The agreement is good in all cases except for the negative discharge at one centimeter distance.

5. Potential Gradients.-E. Warburg ${ }^{1}$ investigated the state of potential at various points between a discharge point and a plane while a current was flowing between them, by means of a platinum probe-point; and the chief feature of his results is the large fall of potential which he found in the immediate neighborhood of the plane this being in some cases one fourth of the potential of the point. Since the maximum distance between the point and the plane used by Warburg in this experiment was only $6 \mathrm{~cm}$. it seemed that with the large distances used in these experiments more trustworthy results could be obtained, since, especially at some distance from the point, the probe would here be less of a disturbing factor.

The probe that was used consisted of a fine platinum wire, $.008 \mathrm{~cm}$. in diameter, soldered to a copper wire, $.025 \mathrm{~cm}$. in diameter, and protruding $1.5 \mathrm{~cm}$. from a finely drawn out glass tube fused about it. The drawn out glass tube formed an elongated tip of a long tapering white pine rod through the center of which ran the wire leading from the probe wire. After leaving the rod the wire was led to a voltmeter through an earthed metal tube. All the observations were made with the probe in the normal line from the discharge point to the plane, and the distance between the plane and the discharge point was 50 centimeters throughout. For each position of the probe the potential was taken for five different voltages of the discharge point.

The results are recorded in Fig. 4. The most salient feature of these curves is the fact that the points for any one distance are collinear. It is found that when the straight lines which best connect the points for the various distances are continued backward till they intersect the axis of the discharge point potential, these intersections are grouped together fairly close except in the case of the short distances where the intersections move towards the origin. It should be noted, however, that it is for these short distances that the probe disturbs the current most seriously. The introduction of the probe diminished the current about I2 per cent. for the $1.5 \mathrm{~cm}$. distance, while the diminution was only 5 per cent. at $7 \mathrm{~cm}$. and fell to 2 per cent. for $15 \mathrm{~cm}$.

${ }^{1}$ Ann. der Phys., IV., 2, p. 299, 1900. 
From the curves in Fig. 4, by the aid of those in Figs. 2 and 3, the potential distance curves drawn in full lines in Fig. 5 were constructed for the two values of the current there indicated. Choosing a certain current, Figs. 2 and 3 give the potentials required to maintain this current for the two kinds of discharge when the distance is $50 \mathrm{~cm}$.; these dischargepoint potentials are then used to obtain from Fig. 4 the corresponding probe potentials for the various distances, which are plotted in Fig. 5.

The curves in Fig. 5 show that the potential gradient between a point and a plane is much more uniform than it was before the current started to flow. There is indeed a rapid falling off of potential near the point similar to that found by Warburg; but this can hardly be called a potential fall as used in the case of vacuum tubes; for here the potential at the nearest part is actually considerably higher than it must be in the static condition before the current begins to

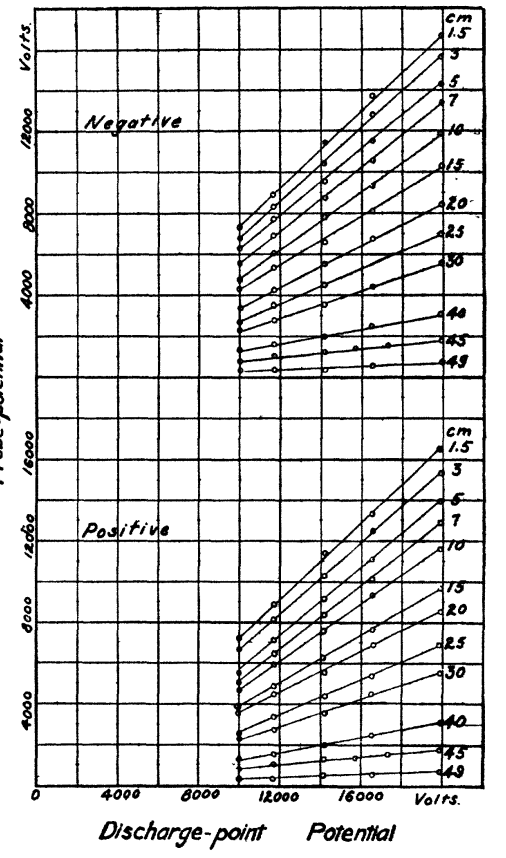

Fig. 4. flow. An idea of the latter case can be obtained by calculation on two concentric spheres.

The curves also show a rapid falling off near the plate; but this is very small as compared with that found by Warburg, and it seems to be, at least for the most part, in the gas and not at the immediate surface of the plate.

There is a method by which one may ascertain the distribution of potential between the point and the plane and avoid the use of the disturbing probe. For a given distance, say $50 \mathrm{~cm}$., there is a certain distribution of potential for a given current. One of the equi-potential surfaces may be replaced by a metal surface and the current will remain unchanged if the same potential difference exists between the point and the surface as before; that is, if the surface introduced be now connected to earth, the potential of the point must be reduced by the previous value of the potential of the surface. The difference between the voltages on the point required to maintain the same current in the two cases gives the potential of the place in the first case before the new surface was in- 
[VoL. XXXIV.

troduced. This can be repeated for various distances and a potentialdistance curve plotted in this way. Supposing that a plane may replace roughly the actual equi-potential surface in question, the desired potentials can be obtained from the potential-current curves for the various distances as given in Figs. 2 and 3. It is only necessary to select a given current and find the point voltages required to maintain this current for the different distances. The differences between these give the desired data.

In using this method of differences, there is an error which may be introduced by the fact that the fall of potential close to the plate for a given current is different for different distances between the point and the plane. As a probe disturbs the conditions least when near the plate,

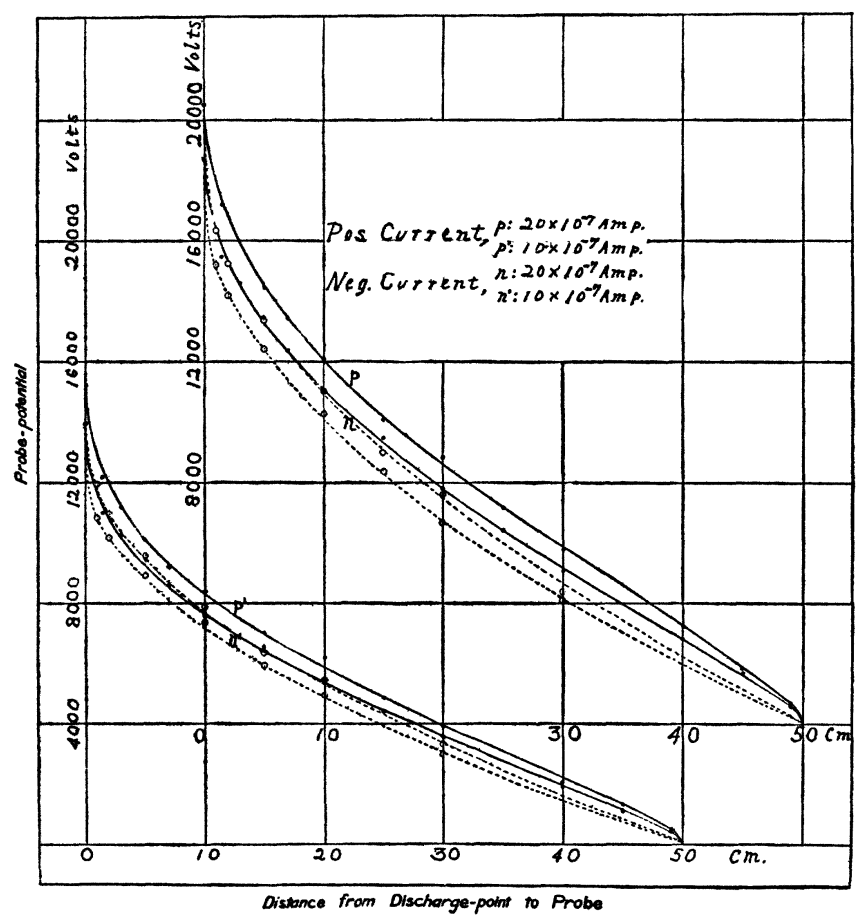

Fig. 5.

a special determination of these drops in potential at the plate was made by the probe method for the different distances and different currents, and corresponding corrections were made in taking the differences of potential in question.

The dotted curves in Fig. 5 were obtained in this way and for the sake of comparison were constructed for the same values of the current as the 
other curves there shown, the upper one being for the positive discharge in each case, as before. The points thus determined are indicated by open circles, while filled circles denote the points obtained by the other method. It is seen that the two methods give results which are quite alike except that the dotted curves fall somewhat below the others.

As no current observations were taken for distances between $30 \mathrm{~cm}$. and $50 \mathrm{~cm}$. this portion of the curves under consideration could not be accurately determined.

The slopes of these curves, that is the value of $d V^{\prime} / d D$ for the various points, may be obtained mechanically with a fair degree of accuracy. But instead of using the mechanical method an empirical relation for $V^{\prime}$ in terms of $D$ was obtained for the curves determined by the probe method, and the slope found by differentiation. A sample set of values of these gradients at different points is given in the following table for the two kinds of discharge, both with a current value of $10 \times \mathrm{IO}^{-7}$ amp. The ratio of these gradients for the various distances is also given in the last column.

\begin{tabular}{r|c|c|c}
\hline$D$ & $\left(\frac{d V^{\prime}}{d D}\right)_{+}$ & $\left(\frac{d V^{\prime}}{d D}\right)_{-}$ & Ratio. \\
\hline 1 & -623 & -416 & 1.50 \\
2 & -457 & -385 & 1.19 \\
5 & -388 & -333 & 1.17 \\
10 & -296 & -270 & 1.10 \\
15 & -251 & -226 & 1.11 \\
20 & -216 & -194 & 1.12 \\
30 & -171 & -153 & 1.12 \\
40 & -181 & -163 & 1.12 \\
45 & -238 & -212 & 1.12 \\
\hline
\end{tabular}

It is noticed that the values of the gradients diminish as one recedes from the point, reach a minimum, and then increase again near the plate. The gradient for the positive discharge is always larger than the corresponding one for the negative discharge. Neglecting the short distances, the ratio of the two gradients is practically the same at all points and for all values of the current. The average value of the ratios for currents between $5 \times 10^{-7}$ and $20 \times 10^{-7} \mathrm{amp}$. was found to be I.II. The ratio for a distance of one centimeter was much larger in every case.

The potential gradient at any point depends upon the value of the current flowing, and it was found that the ratio of the gradients at any point for any two given currents is practically the same for both kinds of discharge and for all places between the point and the plane. For the currents $20 \times 10^{-7}$ and $5 \times 10^{-7}$ amp., the average ratio found for the 
gradients was 2.05; for currents 20 and Io of the same units, the average ratio was 1.43 , while that for currents 20 and 15 , it was I.I6. It is seen that these ratios are but slightly in excess of the square root of the ratios of the corresponding currents.

If $i$ denote current density along the normal where the gradients were determined, $u$ the ionic velocity for unit gradient, and $n$ the electric density, then

$$
i=u n \frac{d V^{\prime}}{d D}
$$

or,

$$
\frac{d V^{\prime}}{d D}=\frac{i}{u n}=K \sqrt{i}
$$

by the above results, remembering that Warburg ${ }^{1}$ showed that the total current is proportional to the central current density. Hence $[n \infty \sqrt{i}]$; or the density of the electric charge at all points and for currents of both signs is proportional to the square root of the current flowing.

For the same current value,

$$
\frac{\left(u_{-}\right)\left(n_{-}\right)}{\left(u_{+}\right)\left(n_{+}\right)}=\frac{\left(\frac{d V^{\prime}}{d D}\right)_{+}}{\left(\frac{d V^{\prime}}{d D}\right)_{-}}=\text {I.II }
$$

Hence,

$$
n_{-}=\mathrm{I} . \mathrm{II}\left(n_{+}\right)\left(\frac{u_{+}}{u_{-}^{-}}\right) \text {. }
$$

The ratio I.I I may depend upon the ratio $\left(u_{+} / u_{-}\right)$, as it was determined for one value only of the latter, i. e., that existing in the room air of average humidity. The relation shows however that for the same current of both signs the electric density is not greatly different, and if the value I.I I holds for moist air, ${ }^{2}$ the two values of $n$ would be exactly equal.

6. Summary.-The starting potential and the currents for different voltages have been determined for a discharge from a point to a plane for distances up to $100 \mathrm{~cm}$. between the latter. The distribution of potential in the air has also been investigated.

It has been found that the starting potential for the short distances between the point and the plane increases rapidly with increasing distances, while at larger distances the increase becomes small, the starting potential appearing to approach a constant value for very large distances.

The currents increase rapidly with the voltage while the distances

1 Loc. cit.

2 J. Zeleny, Phil. Trans., A. I95, p. 2 10, 1900. 
are small but increase very slowly with increasing voltage for the larger distances.

The potential gradients along the normal from a discharge point to a plane decrease rapidly as one recedes from the point, pass through a minimum and again increase near the plate.

The gradients at any point have been found to be proportional to the square root of the current flowing. Omitting the shortest distances the potential gradient for a positive current of given value bears a constant ratio to the potential gradient for the negative current of the same value.

In conclusion I wish to acknowledge my indebtedness to Professor J. Zeleny for the kindly interest he has shown in the progress of the experiments and for his helpfulness in the preparation of this paper.

Physical Laboratory, University of Minnesota,

August 30, 19r r. 